\title{
Sonographische Darstellbarkeit der Intervertebralgelenke an der Brust- und Lendenwirbelsäule des Pferdes
}

\author{
Renate Reisinger und Christian Stanek \\ Aus der Klinik für Orthopädie bei Huf- und Klaventieren Klinisches Department $\bigvee$ für Kleintiere und Pferde Veterinärmedizinische Universität Wien
}

\begin{abstract}
Zusammenfassung
An 7 Rückenpräparaten und 12 lebenden Pferden wurden die Intervertebralgelenke der Brust- und Lendenwirbelsäule, mit Schwerpunktbereich Th10-16, in transversaler und sagittaler Ebene sonographisch untersucht. An der mittleren Brustwirbelsäule war die Darstellung der Gelenkfortsätze und Gelenkspalten, aufgrund der tiefen anatomischen Lage und Winkelung, nur in geringem Ausmaß möglich. Dennoch kann die Ultraschalluntersuchung in dieser Körperregion zur Abklärung von knöchernen Konturveränderungen empfohlen werden.
\end{abstract}

Schlüsselwörter: Sonographie, Pferd, Rücken, Wirbelsäule, Intervertebralgelenke

Sonographic examination of the intervertebral joints of the thoracic and lumbar spine of the horse.

The intervertebral joints of the thoracic and lumbar spine of 7 cadaver specimens and 12 horses were examined ultrasonographically in transversal and sagittal planes with special attention paid to Th 10-16. The depiction of the articular processes as well as the joint spaces of the middle of the thoracic spine was very poor due to the anatomic position and angulation. Nevertheless, ultrasonography of this body region can be recommended to differentiate changes in the osseous contour.

Keywords: ultrasonography, horse, back, spine, intervertebral joints

\section{Einleitung}

Die Intervertebralgelenke (IVG) der Brust- und Lendenwirbelsäule des Pferdes sind aufgrund ihrer tiefen Lage am Rücken einer sonographischen Untersuchung schlecht zugänglich. Dies ist bedingt durch die Lage seitlich am Wirbel, die sich von kranial nach kaudal ändernde Richtung der Gelenkfacetten und auch durch die Überlagerung durch die Rückenmuskulatur. Nach der anatomischen Situation erscheint es dennoch möglich, die Oberfläche der das Intervertebralgelenk bildenden Knochen, den Gelenkspalt sowie benachbarte Muskulatur, Band- und Knochenstrukturen darzustellen (Reisinger 2003). Der Literatur sind zu dieser Frage nur wenige Angaben zu entnehmen.

Denoix (1999) beschreibt die erfolgreiche sonographische Untersuchung der IVG im Bereich der kaudalen Brustwirbelsäule und Lendenwirbelsäule. Gundel et al. (1998) hingegen berichten von schlechter Darstellbarkeit. Nach von Rotz et al. (2002) können die IVG und die Rippen-Wirbelgelenke sonographisch nicht dargestellt werden. Diese Divergenzen und Fortschritte in der Arthrosonographie waren Anlass, die Darstellbarkeit des Gelenkspaltes der IVG, der Processus articulares mit einem $3,5 \mathrm{MHz}-S c h a l l k o p f$ zunächst an Leichenteilen und folgend an rückengesunden und an Rückenproblemen leidenden Pferden zu prüfen.

\section{Material und Methode}

Für die sonographischen Untersuchungen wurde das Gerät SONOLINE SIENNA (Fa. Siemens) verwendet. Zur Darstel- lung des Ligamentum supraspinale, der dorsalen Knochenkontur der Dornfortsätze und der langen Rückenmuskulatur wurde ein $7,5 \mathrm{MHz}$ Linearschallkopf mit Vorlaufstrecke eingesetzt. Die Intervertebralgelenke wurden mit einem $3,5 \mathrm{MHz}$ Konvexschallkopf ohne Vorlaufstrecke mit gegebener größerer Eindringtiefe untersucht.

Die Grundlagen zur sonographischen Untersuchung wurden an 7 Rückenpräparaten (4 Warmblüter, 1 arabisches Vollblut, 2 Ponies; 2 Stuten, 4 Hengste, 1 Wallach; 1,5 bis 24 Jahre) erarbeitet. Bei sechs dieser Präparate wurden zur Identifikation sonographischer Abbildungen farbliche Markierungen per Injektionsnadel ultraschallgeleitet appliziert. Im Rahmen der angeschlossenen Sektion konnten diese den anatomischen Strukturen zugeordnet werden, zum Beispiel den Gelenkspalten der Intervertebralgelenke. Der Tötungsgrund dieser Pferde war ein anderer als eine Erkrankung des Rükkens.

Die sonographische Untersuchung lebender Pferde erfolgte an neun klinikeigenen Pferden (7 Warmblutpferden, 1 englisches Vollblut, 1 Pony; 1 Stute, 2 Hengste, 6 Wallachen; 1,5 bis 20 Jahre) der Veterinärmedizinischen Universität, sowie an drei Rückenpatienten ( 3 Warmblutpferden; 1 Stute, 2 Wallachen; 9 bis 12 Jahre). Die Pferde wurden dafür in einen Zwangsstand verbracht.

Die Untersuchung der IVG erfolgte von dorsal und wurde systematisch, in Richtung des Haarstriches, von kranial nach kaudal und von median nach paramedian durchgeführt. Die 
sonographische Orientierung erfolgte an den palpierbaren Dornfortsätzen (DFS). Die Darstellbarkeit der Intervertebralgelenke zwischen Th10/11, Th12/13, Th14/15, Th16/17 und Th18/L1, sowie L1/2, L3/4 und L5/6 wurde jeweils gesondert beurteilt. Es wurden das Penetrationsvermögen, die Darstellbarkeit der Procc. articulares craniales et caudales, sowie die Darstellbarkeit des Gelenkspaltes beurteilt.

Jedes IVG wurde sowohl transversal als auch longitudinal dargestellt. Bei der transversalen Untersuchung wurde die Schallkopfposition so gewählt, dass je nach untersuchter Körperseite die laterale Knochenkontur des DFS im linken bzw. rechten Bildrand erkennbar war. In dieser Weise wurden die linke und rechte Rückenseite von kranial nach kaudal (Th10 - L6) untersucht (Abb. 1).

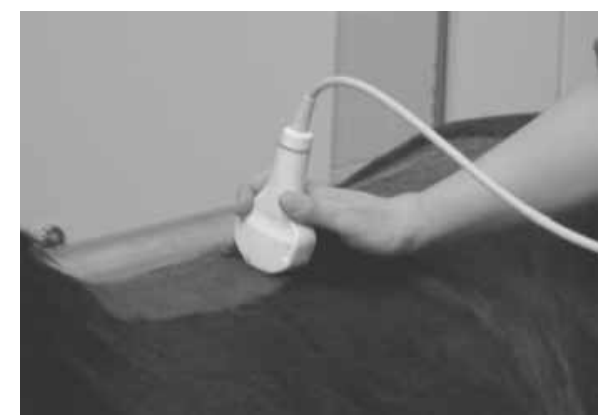

Abb 1 Ausrichtung des Schallkopfes bei transversaler Darstellung der untersuchten Region.

Positioning of the probe during transversal evaluation of the examined region.

Bei der longitudinalen (sagittalen) Untersuchung wurde der Schallkopf longitudinal auf einem DFS ausgerichtet und dann ca. eine Schallkopfbreite nach paramedian versetzt. Von dieser Position aus wurde der Schallkopf in ca. $30^{\circ}$ gegen den DFS gerichtet und dann in einem Schwenk von median nach lateral über die Knochenkonturen der Wirbel geführt. Nach einem jedem solchen Schwenk wurde der Schallkopf um eine Schallkopflänge nach kaudal versetzt, bzw. auch entlang der dargestellten Strukturen nach kaudal geführt (Abb. 2).

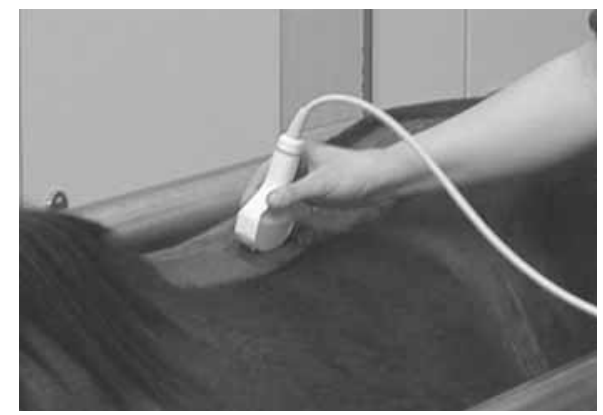

Abb 2 Ausrichtung des Schallkopfes bei sagittaler Darstellung der untersuchten Region.

Positioning of the probe during sagital evaluation of the examined region.

\section{Ergebnisse}

Die Weichteilgewebestrukturen und Knochenoberflächen der Brust- und Lendenwirbelsäule waren sonographisch sowohl an Kadavern als auch an lebenden Pferden gut darstellbar und vergleichbar. Korrespondierende anatomische Gefrierschnitte und die Sektionsergebnisse der Rückenregion erga- ben eine gute Übereinstimmung mit den sonographischen Befunden.

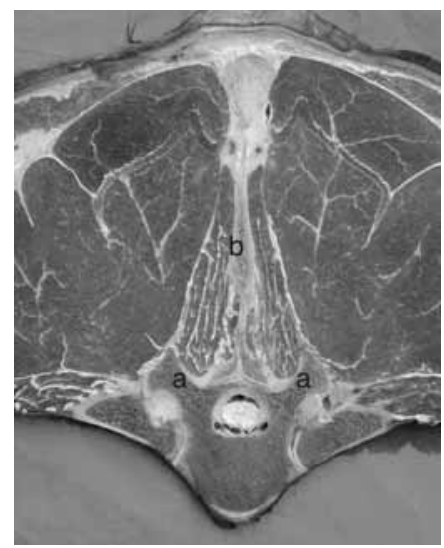

Abb 3a Transversaler Gefrierschnitt durch die mittlere Brustwirbelsäule eines 16jährigen Warmblutwallachs. (a) Zitzenfortsätze, (b) Dornfortsatz

Transversal section through a frozen specimen in the middle thoracic spine of a 16 years old Warmblod gelding. (a) Proc. mamillaris, (b) Proc. spinosus

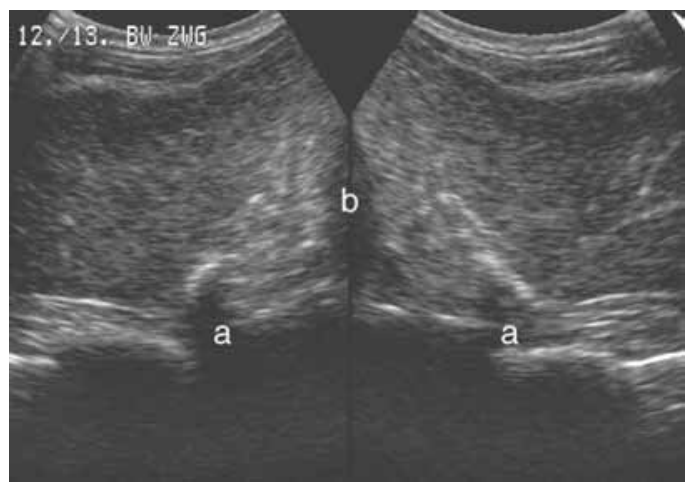

Abb 3b Transversales Doppelbildsonogramm der IVG in Höhe des 12. Brustwirbels eines 23iährigen Isländerwallachs (Präparat). Anhand des Doppelbildes lässt sich sehr gut die Symmetrie der Gelenk- bzw. Zitzenfortsätze (a) überprüfen. Der Dornfortsatz (b) befindet sich in der Mittellinie.

Sonographic double projection in a transversal view of the facet joints of the 12th thoracic vertebral body of a 23 years old lcelandic Horse gelding (specimen). On the basis of the double projection the symmetry of the mamillar and articular processes (a) can be evaluated. The spinous process is found in the midline (b

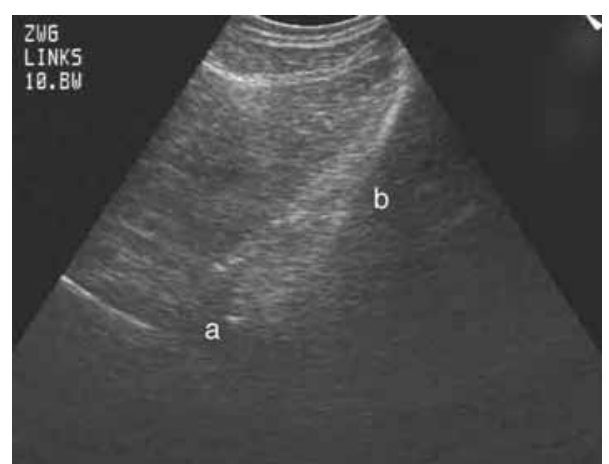

Abb 3c Transversales Sonogramm des linken IVG in Höhe des 10. Brustwirbels eines 12jährigen Hollsteiner Wallachs (lebendes Pferd). (a) Zitzenfortsatz, (b) Dornfortsatz

Transversal sonogram of the left facet joint of the 10th thoracic vertebral body of a 12 years old Hollstein gelding (living horse). (a) Proc. mamillaris, (b) Proc. spinosus 
Zur Orientierung wurde die dorsale Kontur der Dornfortsätze verwendet, welche sich sowohl in sagittaler als auch transversaler Richtung als stark echogene gleichmäßige Linie darstellte. Individuelle Abweichungen der dorsalen Kontur traten auf. Das Ligamentum supraspinale, welches den Dornfortsätzen dorsal aufliegt, ist gut darstellbar, es zeigt bei sagittaler Schallkopfausrichtung deutlich längs ausgerichtete Faserbündel. Je nach Abstand und Form der Dornfortsätze ließen sich Fasern vom Lig. supraspinale bis in den Interspinalraum verfolgen. Transversal zeigt sich das Band als rundlich bis querovale Struktur. Die Rückenmuskulatur stellte sich, gegenüber den umgebenden Faszien und Septen, als geringer echogen dar. Die Mm. multifidi ließen sich eindeutig vom M. longissimus dorsi differenzieren. Die Mm. multifidi sind deutlich von Septen durchzogen und liegen, eine dreieckige Kontur bildend, den Dornfortsätzen lateral an.

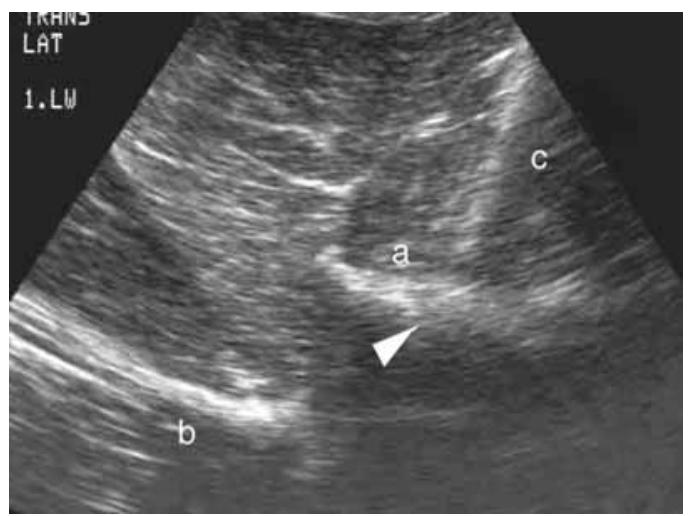

Abb 4 Transversales Sonogramm des linken IVG in Höhe des 1. Lendenwirbels einer 8jährigen Warmblutstute (Präparat). (a) knöchernes Plateau welches durch die Procc. articulares craniales et caudales gebildet wird, (b) Proc. transversus bzw. Lig. intertransversarium, (c) Position des Processus spinosus. Der Pfeil markiert einen echoarmen Bereich in dem stark echogenen Plateau, welche die Region des Gelenkspaltes des IVG darstellt.

Transversal sonogram of the left facet joint of the 1st lumbar vertebral body of a 8 years old Warmblod mare (specimen). (a) bony plateau built by the cranial and caudal articular processes, (b) proc. transversus resp. lig. intertransversarium, (c) position of the spinous process. The arrow marks the less echogen area in the strong echogen plateau, which represents the facet joint space.

Vom 10.-16. Brustwirbel war die sonographische Darstellung der Gelenkfortsätze, sowohl am Präparat als auch am lebenden Pferd, nur in geringem Ausmaß möglich, die Gelenkspalten zudem wegen der annähernd horizontalen Ausrichtung der Gelenkflächen nicht zweifelsfrei darstellbar. Deutlich erkennbar waren jedoch die Procc. mamillares. Im transversalen Schallbild hob sich deren Knochenkontur im Bereich der Dornfortsatzbasis nach lateral "astförmig" ab (Abb. 3a, $3 b, 3 c)$.

Am Präparat ergab sich eine bessere Darstellbarkeit der Gelenkfortsätze und teilweise auch der Gelenkspalten (Abb. 4) ab dem 16. Brustwirbels nach kaudal. Bei transversaler Ultraschalldarstellung an der kaudalen Brustwirbelsäule und an der Lendenwirbelsäule stellten sich die gelenkbildenden kranialen und kaudalen Gelenkfortsätze als Plateau dar, welches sich deutlich von den Dornfortsätzen und den Rippen bzw. den Querfortsätzen der beteiligten Wirbel abgrenzte (Abb. 5a, 5b, 5c). Die anatomische Grundlage dafür bilden die laterale Kontur der Dornfortsatzbasis des kaudalen, an

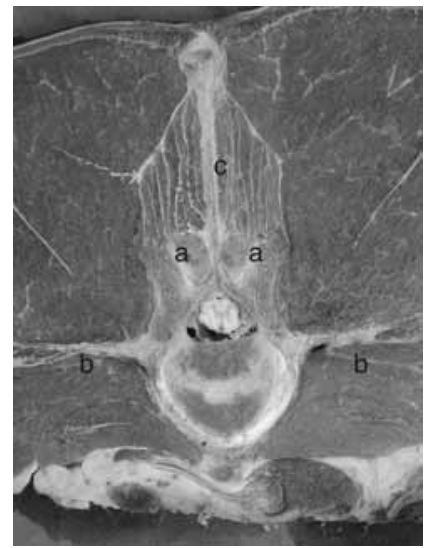

Abb 5a Transversaler Gefrierschnitt durch die Lendenwirbelsäule eines 16jährigen Warmblutwallachs. (a) knöchernes Plateau welches durch die Procc. articulares craniales et caudales gebildet wird, (b) Proc. transversus bzw. Lig. intertransversarium, (c) Position des Processus spinosus

Transversal section through a frozen specimen in the lumbar spine of a 16 years old Warmblod gelding. (a) bony plateau built by the cranial and caudal articular processes, (b) proc. transversus resp. lig. intertransversarium, (c) position of the spinous process

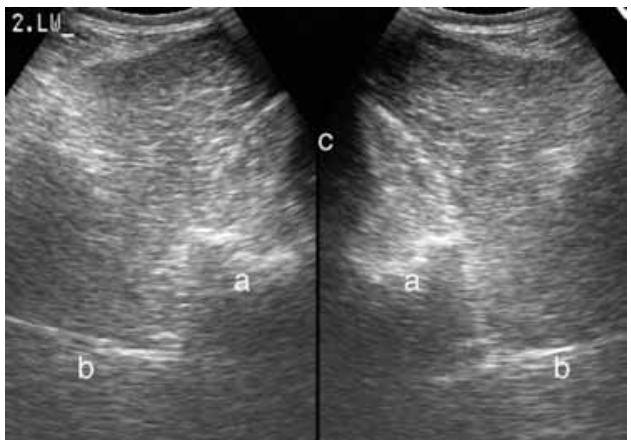

Abb 5b Transversales Doppelbildsonogramm im Bereich des 2 Lendenwirbels eines 13jährigen Warmblutwallachs (lebendes Pferd). (a) knöchernes Plateau welches durch die Procc. articulares craniales et caudales gebildet wird, (b) Proc. transversus bzw. Lig. intertransversarium, (c) Position des Processus spinosus Sonographic double projection in a transversal view of the facet joints of the 2nd lumbar vertebral body of a 13 years old Warmblod gelding (living horse). (a) bony plateau built by the cranial and caudal articular processes, (b) proc. transversus resp. lig. intertransversarium, (c) position of the spinous process.

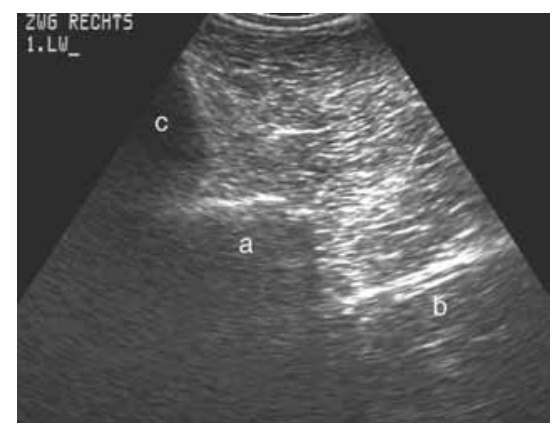

Abb 5c Transversales Sonogramm des rechten IVG in Höhe des 1. Lendenwirbels eines 12jährigen Warmblutwallachs (lebendes Pferd). (a) knöchernes Plateau welches durch die Procc. articulares craniales et caudales gebildet wird, (b) Proc. transversus bzw. Lig. intertransversarium, (c) Position des Processus spinosus Transversal sonogram of the right facet joint of the 1 st lumbar vertebral body of a 12 years old Warmblod gelding (living horse). (a) bony plateau built by the cranial and caudal articular processes, (b) proc. transversus resp. lig. intertransversarium, (c) position of the spinous process. 
der Gelenkbildung beteiligten Wirbels, nach lateral anschlieBend der kaudale Gelenkfortsatz des beteiligten kranialen Wirbels. Dem Intervertebralgelenkspalt schließt nach lateral der kraniale Gelenkfortsatz des beteiligten kaudalen Wirbels an (Abb. 5a). Die Articulationes processuum articularium (IVG) bilden einen schmalen Gelenkspalt, welcher sich bei der Ultraschalluntersuchung als eine kleine echoarme Zone auf dem sich stark echogen darstellenden Knochenplateau zeigen kann.

Im Rahmen der sagittalen Untersuchung wurde die laterale Fläche der Dornfortsätze in einem Schwenk von median nach paramedian abgetastet. Die tiefste Stelle bildete die Dornfortsatzbasis bzw. die Gelenkfortsätze. Nach lateral schlossen sich als markante knöcherne Erhebungen die Procc. mamillares (Abb. 6a, 6b) an. Diese sind an der mittleren Brustwirbelsäule durch ihre nach kranial gerichtete "schanzenartige" Kontur deutlich zu identifizieren. An der

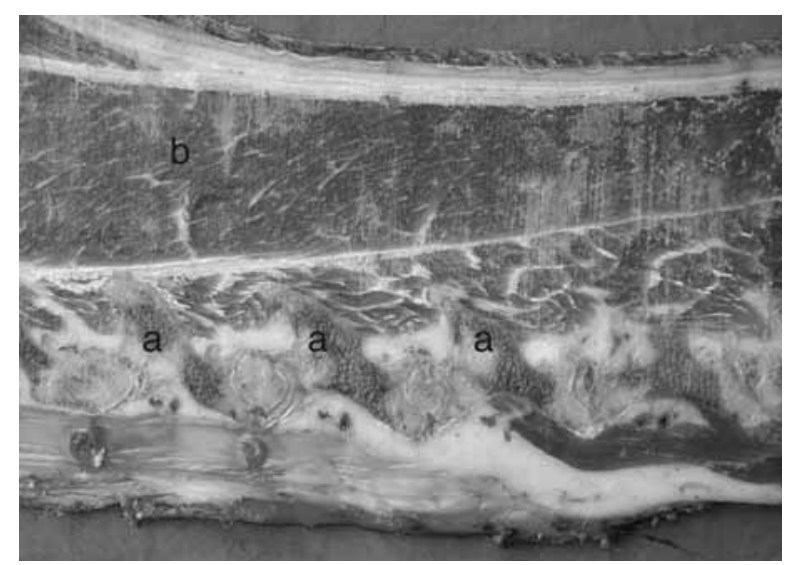

Abb 6a Sagittaler paramedianer Gefrierschnitt durch die mittlere Brustwirbelsäule eines 24jährigen Islandwallachs. (a) Procc.mamillares, (b) M. longissimus dorsi

Sagital paramedian section through a frozen specimen in the middle thoracic spine of a 24 years old Icelandic Horse gelding.

(a) Procc. mamillares, (b) M. longissimus dorsi

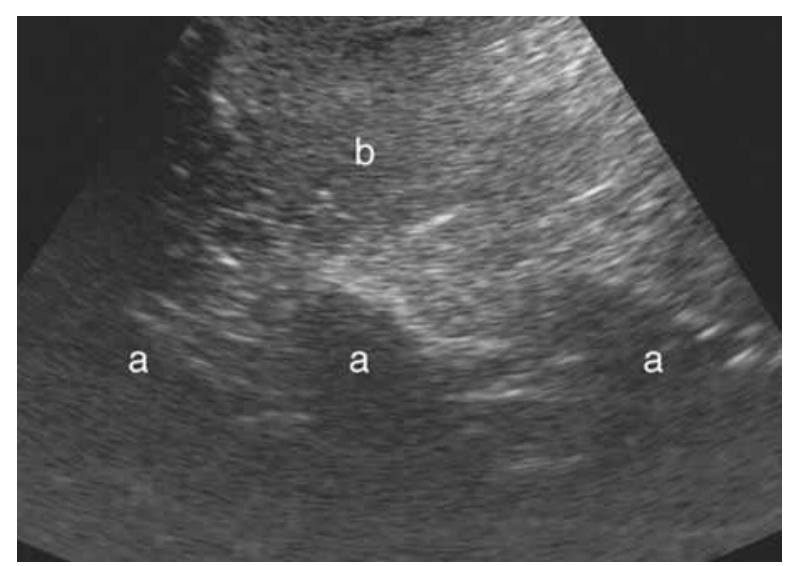

Abb 6b Sagittales Sonogramm der Procc. mamillares der Brustwirbelsäule eines 24jährigen Lipizzanerhengstes (Präparat). Die nach kranial "schanzenartige" Knochenoberfläche der Procc. mamillares (a) hebt sich deutlich vom Muskelgewebe ab, M. Iongissimus dorsi (b).

Sagital sonogram of the thoracic mamillar processes of a 24 years old Lipizzan stallion (specimen). The mamillar processes (a), shapped like a "take-off platform" in cranial direction, are well separated from the muscle tissue, M. Longissimus dorsi (b).
Lendenwirbelsäule imponieren die Procc. mamilloarticulares mit von kranial nach kaudal wellenartiger Kontur (Abb. 7a, 7b). Am Präparat waren die Gelenkspalten der IVG an der mittleren Brustwirbelsäule mit dieser Technik nicht, und an der kaudalen Brust- bzw. Lendenwirbelsäule nicht zweifelsfrei darstellbar.

Am lebenden Pferd war es vom 10. Brustwirbel bis zum 6 . Lendenwirbel, sowohl in transversaler als auch sagittaler Ebene, nicht möglich, Gelenkspalten der IVG zweifelsfrei zu erkennen.

Ein Störfaktor bei der Untersuchung am lebenden nicht sedierten Pferd sind die Muskelkontraktionen bzw. der Muskeltonus. Bei angespannter Rückenmuskulatur vermindert sich die Kontaktfläche des Konvexschallkopfes und damit auch die Darstellbarkeit der zu untersuchenden Strukturen.

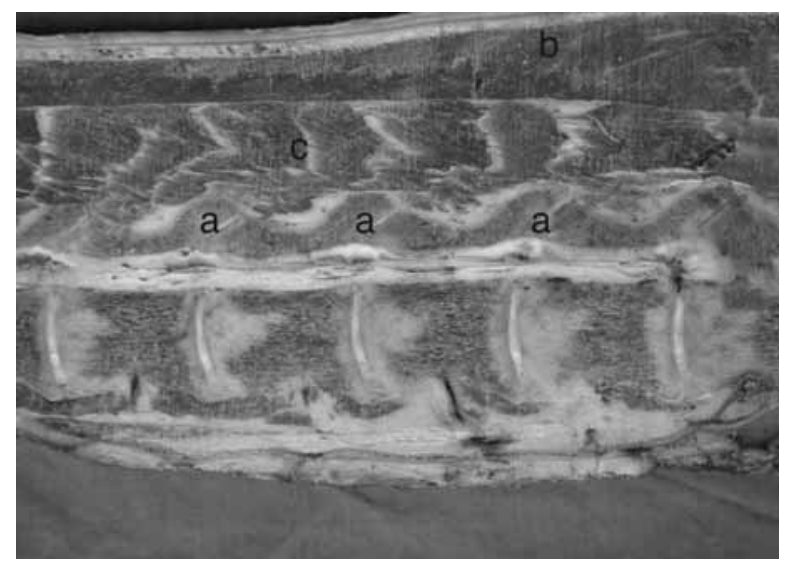

Abb 7a Sagittaler paramedianer Gefrierschnitt durch die Lendenwirbelsäule eines 24jährigen Islandwallachs. (a) Procc. articulares crann. et caudales, (b) M. longissimus dorsi, (c) Mm. multifidi

Sagital paramedian section through a frozen specimen in the lumbar spine of a 24 years old Icelandic Horse gelding. (a) Procc. articulares crann. et caudales, (b) M. longissimus dorsi, (c) Mm. multifidi

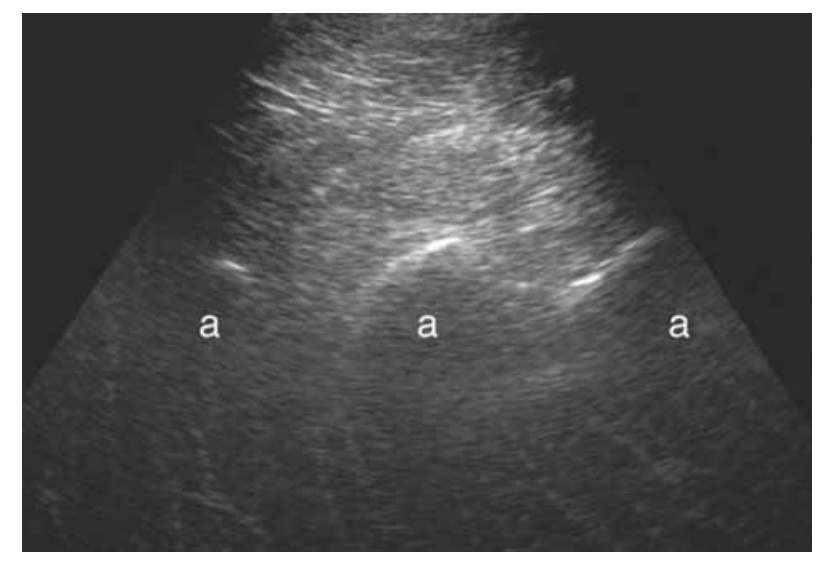

Abb 7b Sagittales Sonogramm der Procc. articulares crann. et caudd. der Lendenwirbelsäule eines 20jährigen Traberwallachs (lebendes Pferd). (a) Procc. articulares crann. et caudales

Sagital sonogram of the cranial and caudal articular processes of the lumbar spine of a 20 years old Standardbred gelding (living horse). (a) Procc. articulares crann. et caudales 


\section{Diskussion}

Die schwierige und technisch aufwendige röntgenologische Darstellbarkeit der Wirbelkörper mit den dort gelegenen Fortsätzen rechtfertigt die Suche nach alternativen Methoden zur Untersuchung. Denoix (1999) beschrieb die sonographische Darstellung der Gelenkfortsätze und Intervertebralgelenke der kaudalen Brustwirbelsäule und der Lendenwirbelsäule. $\mathrm{Er}$ erwähnte die Doppelbilddarstellung zur Überprüfung der Gelenksymmetrie und die ultraschallunterstützte periartikuläre Injektion. Die auf einer latero-lateralen Röntgenaufnahme übereinander projizierten Gelenke, können mit einem Ultraschallgerät einzeln untersucht und mittels Doppelbild auf ihre Symmetrie hin beurteilt werden. Gundel et al. (1998) und von Rotz et al. (2002) stellten bei ihren sonographischen Untersuchungen des Pferderückens fest, dass die Procc. articulares bzw. mamilloarticulares zwar darzustellen, jedoch die Beurteilung der Intervertebralgelenke nicht möglich sei. Von Denoix (1999) und Gundel et al. (1998) wurden keine Angaben über die transversale und sagittale Darstellbarkeit der Gelenkfortsätze und der Gelenkspalten an der mittleren Brustwirbelsäule, bzw. die sagittale Untersuchung an der kaudalen Brustwirbelsäule und Lendenwirbelsäule gemacht. Von Rotz et al. (2002) beschreiben die Formveränderungen der Gelenkfortsätze in der transversalen Abbildung von der mittleren Brustwirbelsäule bis zur Lendenwirbelsäule.

In dieser Studie wurde zur Darstellung der Gelenkfortsätze bzw. der Intervertebralgelenke der Pferderücken vom kaudalen Widerrist bis Lendenwirbelsäule (Th10-L6) sonographisch untersucht.

Wegen der annähernd horizontalen Ausrichtung der Intervertebralgelenkflächen von kranial bis in den Bereich der mittleren Brustwirbelsäule, war es in unserer Studie selbst am Präparat nicht möglich, einen Gelenkspalt zu identifizieren. Aufgrund der horizontalen Ausrichtung wäre es zwar naheliegend, die Schallkopfposition nach lateral zu verlagern, um so bessere Einsicht auf den Gelenkspalt zu erhalten, aber dabei wird einerseits die zu durchdringende Muskelmasse zu groß und andererseits überlagern knöcherne Strukturen wie die Procc. mamillares und proximalen Rippenanteile die IVG. Mit Hilfe der Doppelbilddarstellung kann in transversaler Schallebene die Symmetrie der rechten und linken Knochenstrukturen (Gelenkfortsätze, Zitzenfortsätze) überprüft werden. Eine Asymmetrie kann einen Hinweis auf degenerative Knochenveränderungen geben. Im sagittalen Ultraschallbild können die hintereinander folgenden Knochenstrukturen einer Seite verglichen werden. Ein Apparat mit Panoramabildfunktion kann dafür zum Einsatz kommen. Von Rotz et al. (2002) zeigen Panoramabilder longitudinal der dorsalen Dornfortsatzkonturen und transversal der Rücken-Weichteilstrukturen. Wegen der Anschaffungskosten wird ein solches Ultraschallgerät aber eher größeren Kliniken vorbehalten bleiben. Im Bereich der mittleren Brustwirbelsäule ist zudem eine detaillierte röntgenologische Darstellung der IVG möglich (Butler 2001, Butler et al. 2000, Reisinger 2003, Reisinger und Stanek 2005).

An der kaudalen Brustwirbelsäule beginnen sich die IVG vertikal auszurichten. Darum werden die Gelenkspalten der Ultraschalluntersuchung von dorsal zugänglich. Röntgenologisch muss in dieser Region jedoch aufgrund der Weichteil- masse die latero-laterale Röntgentechnik angewendet werden, wodurch sich die IVG der rechten und linken Wirbelsäulenseite überlagern (Butler 2001, Butler et al. 2000, Jeffcott 1975, 1978, 1985 Ueltschi 2001, Weaver et al. 1999). Am Präparat waren bei transversaler sonographischer Darstellung Gelenkspalten teilweise erkennbar, am lebenden Pferd war deren zweifelsfreie Abbildung jedoch nicht möglich. Es könnte am vorherrschenden Muskeltonus der Rückenmuskulatur gelegen sein. Je nach Durchdringbarkeit des Gewebes, war es an der kaudalen BWS- und LWS-Region möglich, mittels Doppelbilddarstellung wiederum die Symmetrie der Knochenstrukturen bzw. der Gelenkfortsätze zu überprüfen. Dies ist vor allem ab L3/L4 von Interesse, da sich dieser Bereich der röntgenologischen Untersuchung von lateral entzieht. Bei laterolateralem Strahlengang überlagern die Darmbeinschaufeln die kaudalen Anteile der Lendenwirbelsäule. Zur röntgenologischen Darstellung wären ventro-dorsale Aufnahmen in Allgemeinanästhesie in Rückenlage nötig (Butler 2001, Butler et al. 2000, Jeffcott 1975, 1978, 1985, Ueltschi 2001, Weaver et al. 1999). Hier bietet die Ultraschalluntersuchung in der kaudalen Lendenwirbelregion transkutan von dorsal eine gewisse Alternative.

Die Ultraschalluntersuchung der Brust- und Lendenwirbelsäule des Pferdes ermöglicht somit durchaus die Erhebung zusätzlicher Befunde zu einer Röntgenuntersuchung. Obwohl die IVG im Bereich der mittleren BWS über dem Lungenfeld (Bereich Th 9 - Th 16) röntgenologisch sehr gut darstellbar sind (Reisinger 2003, Reisinger und Stanek 2005), kann die Symmetrie der knöchernen Strukturen sonographisch besser beurteilt werden. Sie ermöglicht auch die separate Darstellung der Gelenkfortsätze und Symmetriebeurteilung. In unserer Studie war einwandfreie Darstellbarkeit der IVG am lebenden Pferd nicht zweifelsfrei möglich. Ähnlich beschreiben dies Gundel et al. (1998) und von Rotz et al. (2002).

Diese Untersuchungen gaben jedoch auch zu erkennen, dass je nach Körperbau und Typ des Pferdes sowie entsprechendem Ultraschallgerät, die sonographische Untersuchung der mittleren Brustwirbelsäule von diagnostischer Relevanz sein kann. Die Sonographie bietet Informationen über die Symmetrie der sich gegenüber liegenden knöchernen Strukturen. Asymmetrien bzw. einseitige knöcherne Zubildungen würden Hinweise auf pathologische Prozesse darstellen.

Die Möglichkeiten der sonographischen Untersuchungen in sagittaler und transversaler Ebene sollten im Rahmen einer detaillierten Untersuchung eines Rückenpatienten, zur Beurteilung der periartikulären Strukturen, nicht außer Acht gelassen werden. Sie umfassen nicht nur die Darstellung der Proc. articulares und mamilloarticulares, sondern auch die der Procc. spinosi und der sie verbindenden Bandanteile.

\section{Literatur}

Butler J. (2001): Persönliche Mitteilung

Butler J. A., Colles C., Dyson S., Kold S. E. und Poulus P. W. (2000): Clinical radiology of the horse. 2. ed. Blackwell Scientific Publications, Oxford, 355-398

Denoix J.-M. (1999) Ultrasonographic evaluation of back lesions. Veterinary Clinics of North America: Equine Pract. 15, 131-159. 
Gundel M., Schmucker N., Budde K., von Rotz A., Schatzmann U. und Meier H. (1998): Die sonographische Untersuchung am Rücken des Pferdes: Grundlagen und Untersuchungstechnik an ausgewählten Strukturen des Pferderückens. Pferdeheilkunde 14, 322-332

Jeffcott L. B. (1975): The diagnosis of diseases of the horse's back. Equine Veterinary Journal 7, 69-78

Jeffcott L. B. (1978): Klinische und röntgenologische Diagnostik der Wirbelsäulenerkrankungen des Pferdes. Der praktische Tierarzł 4. 272-28.

Jeffcott L. B. (1985): The examination of a horse with a potential back problem. Proc. of the 31 st annual convention of the american association of equine practitioners. Toronto. 285-297

Reisinger R. (2003): Röntgenologische und sonographische Darstellbarkeit der Intervertebralgelenke der Brust- und Lendenwirbelsäule des Pferdes. Diss. med. vet., Veterinärmedizinische Universität Wien

Reisinger R. und Stanek C. (2005): Vergleich der $20^{\circ}$ und $30^{\circ}$ Technik zur radiologischen Darstellung der Intervertebralgelenke an der Brustwirbelsäule des Pferdes. Pferdeheilkunde, im Druck
Ueltschi G. (2001): Radiologie und Szintigraphie der Wirbelsäule Proc. 7. Kongress für Pferdemedizin und -chirurgie, Genf, Schweiz, 47-51

von Rotz A., Frey R., Friess A. E., Schatzmann U. und Meier $H$. (2002): Die sonographische Untersuchung des Pferderückens. Pferdeheilkunde 18, 155 - 161

Weaver M. P., Jeffcott L. B. und Nowak M. ( 1999): Radiology and Scintigraphy, In: Veterinary Clinics of North America: Equine Practice 15, 113-129

\section{Dr. Renate Reisinger}

Veterinärmedizinische Universität Wien

Klinisches Department V für Kleintiere und Pferde

Klinik für Orthopädie bei Huf- und Klaventieren

Veterinärplatz 1

1210 Wien

renate.reisinger@vu-wien.ac.at

\title{
Pferdeheilkunde Forum 2005 - Berliner Fortbildungstage
}

\section{6.-19. Juni 2005, Hilton Berlin und Humboldt-Universität}

\author{
Blockseminare Donnerstag, 16. Juni 2005
}

\section{1 bis $14 \mathrm{Uhr}$}

\section{1 - Der Röntgenleitfaden - Bodo Hertsch}

2 - Rehe - Hartmut Gerhards

3 - Dermatologie - Arthur Grabner

4 - Orthopädischer Hufbeschlag - Christian Stanek

5 - Teamentwicklung und Teamarbeit - Yasmine Bouali

6 - Kardiologische Diagnostik - Heidrun Gehlen und Peter Stadler

7 - Neurologische Diagnostik - Karsten Feige

\section{5-18 Uhr}

8 - Physiologie des Gelenks und Pathologie der OCD - René van Weeren

9 - Bakterielle Toxikosen - Vinzent Gerber

10 - Sichere Narkoseführung - Urs Schatzmann

11 - Aktuelle Forensik - Dietrich Plewa und Hartmut Gerhards

12 - Finanżmanagement und Controlling - Claudia Menke

13 - Sonographische Gelenkdiagnostik - Johannes Edinger

14 - Diagnostische Anästhesien - Astrid B. M. Rijkenhuizen

\section{$18.30 \mathrm{bis} 21 \mathrm{Uhr}$}

15 - Praxismarketing und Praxismanagement - Anne Cathérine Coppens

16 - Doping - Fenner, Gerweck, Plewa, Schatzmann, Schenk, Sluyter

\section{www.pferdeheilkunde.de}

